\title{
Correction to the article: Floer homology and splicing knot complements
}

\author{
EAMAN EFTEKHARY
}

\begin{abstract}
This note corrects the mistakes in the splicing formulas of the paper "Floer homology and splicing knot complements" [1]. The mistakes are the result of the incorrect assumption that for a knot $K$ inside a homology sphere $Y$, the involution on $\widehat{\operatorname{HFK}}(K)$ which corresponds to moving the basepoints by one full twist around $K$ is trivial. The correction implicitly involves considering the contribution from this (possibly non-trivial) involution in a number of places.
\end{abstract}

57M27; 57R58

We first describe the incorrect assumption about the triviality of the involution on $\widehat{\operatorname{HFK}}(K)$ which corresponds to moving the basepoints by one full twist around a knot $K$ inside a homology sphere $Y$. The incorrect assumption that this map is trivial first appears in Subsection 3.3 of "Floer homology and splicing knot complements" [1]. It is then used in Subsection 5.3 to give a simplified matrix presentation of the splicing formula. For the trefoils, the involution map happens to be trivial, and thus the computations of Subsection 6.1 remain unchanged. Some modifications are necessary to the computations of subsection 6.2. Finally, there are some changes that should be made to the matrix presentations in the appendix. The corrected versions of subsections 3.3, 5.3 and 6.2, as well as the corrected form of the appendix appear in this note.

The original form of the splicing formulas, which are based on the aforementioned wrong assumption, are in fact not incorrect. However, the arguments of this paper are not sufficient for showing this and the corrections of this note are thus very crucial. The issue is further discussed in the sequel [2].

The author would like to thank Adam Levine for pointing out the mistake, and for some very helpful discussions.

\section{The involution of $\widehat{\mathrm{HFK}}$}

In Subsection 3.3 of [1], the author considers the isomorphisms $\tau_{\bullet}: \mathbb{H}_{\bullet}(K) \rightarrow \mathbb{H}_{\bullet}(K)$ for $\bullet \in\{0,1, \infty\}$ coming from changing the role of the two punctures $z_{1}$ and $z_{2}$ in 
the Heegaard diagram $\left(\Sigma, \boldsymbol{\alpha}, \boldsymbol{\beta} ; z_{1}, z_{2}\right)$. It was claimed that $\tau_{\bullet}^{2}$ is the identity map. However, the isomorphism $\tau_{\bullet}^{2}$ is in fact the involution map $\varsigma_{\bullet}$ studied by Sarkar [7] and by Hendricks and Manolescu [4], which is not necessarily trivial. This map corresponds to moving the punctures $z_{1}$ and $z_{2}$ one full twist along the knot $K$ and its square is the identity map. The map $\varsigma_{\infty}$ is in general non-trivial, e.g. for the figure-eight knot. Correspondingly, it is not a priori true that

$$
\overline{\mathfrak{f}}_{0}=\tau_{\infty} \circ \mathfrak{f}_{0} \circ \tau_{1}, \overline{\mathfrak{f}}_{1}=\tau_{0} \circ \mathfrak{f}_{1} \circ \tau_{\infty} \text { and } \overline{\mathfrak{f}}_{\infty}=\tau_{1} \circ \mathfrak{f}_{\infty} \circ \tau_{0} .
$$

Without the above inequalities, many of the matrix computations in Subsection 5.3 need significant changes and the computation of Subsection 6.3 should also be modified. Some changes are also necessary in the later parts of the appendix. The revised forms of these parts follow.

Nevertheless, the 3 equalities

$$
\overline{\mathfrak{f}}_{0}=\tau_{\infty} \circ \mathfrak{f}_{0} \circ \tau_{1}, \overline{\mathfrak{f}}_{1}=\tau_{0} \circ \mathfrak{f}_{1} \circ \tau_{\infty} \text { and } \overline{\mathfrak{f}}_{\infty}=\tau_{1} \circ \mathfrak{f}_{\infty} \circ \tau_{0} .
$$

are true for non-trivial reasons that will not be discussed here and are not relevant for the purposes of this paper. A detailed discussion of this claim appears in [2].

\section{Subsection $3.3^{\prime}$ : Some properties of the maps $\mathfrak{f}_{\bullet}(K)$ and $\overline{\mathfrak{f}}_{\bullet}(K)$}

Our first observation is that changing the orientation of the knot $K$, and correspondingly that of $K_{1}$ and $K_{0}$, corresponds to changing the markings $u, v, w$ with $\bar{u}, \bar{v}, \bar{w}$ in Figure 1. Suppose that $\left(\Sigma, \boldsymbol{\alpha}, \boldsymbol{\beta} ; z_{1}, z_{2}\right)$ represents $K_{\bullet}$, meaning that an oriented longitude for $K_{\bullet}$ is constructed from gluing an oriented arc on $\Sigma$ from $z_{1}$ to $z_{2}$ in the complement of $\boldsymbol{\alpha}$ and an oriented arc on $\Sigma$ from $z_{2}$ to $z_{1}$ in the complement of $\boldsymbol{\beta}$. Then $\left(\Sigma, \boldsymbol{\alpha}, \boldsymbol{\beta} ; z_{2}, z_{1}\right)$ is a Heegaard diagram for $-K$ ( the knot $K \boldsymbol{\bullet}$ with the reverse orientation) while $\left(-\Sigma, \boldsymbol{\beta}, \boldsymbol{\alpha} ; z_{2}, z_{1}\right)$ is a Heegaard diagram for $K_{\boldsymbol{\bullet}}$. The chain complexes associated with the above three Heegaard diagrams are identical. Heegaard moves give chain homotopy equivalences

$$
\tau_{\bullet}(K): \widehat{\mathrm{CF}}\left(\Sigma, \boldsymbol{\alpha}, \boldsymbol{\beta} ; z_{1}, z_{2}\right)=\widehat{\mathrm{CF}}\left(-\Sigma, \boldsymbol{\beta}, \boldsymbol{\alpha} ; z_{2}, z_{1}\right) \longrightarrow \widehat{\mathrm{CF}}\left(\Sigma, \boldsymbol{\alpha}, \boldsymbol{\beta} ; z_{1}, z_{2}\right) .
$$

These chain homotopy equivalences induce the isomorphisms

$$
\tau_{\bullet}(K): \mathbb{H}_{\bullet}(K) \longrightarrow \mathbb{H}_{\bullet}(K), \quad \bullet \in\{0,1, \infty\} .
$$

In terms of these isomorphisms

$$
\begin{aligned}
& \overline{\mathfrak{f}}_{0}(K)=\tau_{\infty}(K) \circ \mathfrak{f}_{0}(K) \circ \tau_{1}(K)^{-1}, \\
& \overline{\mathfrak{f}}_{1}(K)=\tau_{0}(K) \circ \mathfrak{f}_{1}(K) \circ \tau_{\infty}(K)^{-1} \text { and } \\
& \overline{\mathfrak{f}}_{\infty}(K)=\tau_{1}(K) \circ \mathfrak{f}_{\infty}(K) \circ \tau_{0}(K)^{-1} .
\end{aligned}
$$


Note however, that the equality $\bar{\theta}(K)=\tau_{\infty}(K) \theta(K) \tau_{0}(K)^{-1}$ is only satisfied for the induced maps from $\operatorname{Ker}\left(\overline{\mathfrak{f}}_{\infty}(K)\right)$ to $\operatorname{Coker}\left(\overline{\mathfrak{f}}_{0}(K)\right)$.

The exactness of the sequences in (3) implies that in appropriate decompositions

$$
\begin{aligned}
& \mathbb{H}_{0}(K)=\frac{\mathbb{H}_{0}(K)}{\operatorname{Ker}\left(\mathfrak{f}_{\infty}(K)\right)} \oplus \operatorname{Ker}\left(\mathfrak{f}_{\infty}(K)\right)=: \mathbb{A}_{\infty}(K) \oplus \mathbb{A}_{1}(K), \\
& \mathbb{H}_{1}(K)=\frac{\mathbb{H}_{1}(K)}{\operatorname{Ker}\left(\mathfrak{f}_{0}(K)\right)} \oplus \operatorname{Ker}\left(\mathfrak{f}_{0}(K)\right)=: \mathbb{A}_{0}(K) \oplus \mathbb{A}_{\infty}(K) \text { and } \\
& \mathbb{H}_{\infty}(K)=\frac{\mathbb{H}_{\infty}(K)}{\operatorname{Ker}\left(\mathfrak{f}_{1}(K)\right)} \oplus \operatorname{Ker}\left(\mathfrak{f}_{1}(K)\right)=: \mathbb{A}_{1}(K) \oplus \mathbb{A}_{0}(K)
\end{aligned}
$$

we have $\mathfrak{f}_{\bullet}(K)=\left(\begin{array}{cc}0 & 0 \\ I_{a_{\bullet}(K)} & 0\end{array}\right)$, where $a_{\bullet}(K)$ denotes the rank of $\mathbb{A} \bullet(K)$ for every $\bullet \in$ $\{0,1, \infty\}$. In this basis we may present the matrices $\tau_{\bullet}(K)$ as

$$
\tau_{\bullet}(K)=\left(\begin{array}{cc}
A_{\bullet}(K) & B_{\bullet}(K) \\
C_{\bullet}(K) & D_{\bullet}(K)
\end{array}\right), \quad \bullet \in\{0,1, \infty\}
$$

The map $B_{0}(K)$ corresponds to the induced map

$$
\tau_{0}(K): \operatorname{Ker}\left(\mathfrak{f}_{\infty}(K)\right) \rightarrow \frac{\mathbb{H}_{0}(K)}{\operatorname{Ker}\left(\mathfrak{f}_{\infty}(K)\right)}
$$

The decomposition $\mathbb{H}_{0}(K)=\mathbb{A}_{\infty}(K) \oplus \mathbb{A}_{1}(K)$ may be modified using a change of basis of the form $P_{X}=\left(\begin{array}{cc}I & 0 \\ -X & I\end{array}\right)$, which does not change the block presentations of the maps $\mathfrak{f}_{\infty}(K)$ and $\mathfrak{f}_{1}(K)$. In the new basis $\tau_{0}(K)$ has the following presentation:

$$
\begin{aligned}
\tau_{0}(K) & =\left(\begin{array}{cc}
I & 0 \\
-X & I
\end{array}\right)\left(\begin{array}{cc}
A_{0}(K) & B_{0}(K) \\
C_{0}(K) & D_{0}(K)
\end{array}\right)\left(\begin{array}{cc}
I & 0 \\
-X & I
\end{array}\right) \\
& =\left(\begin{array}{cc}
A_{0}(K)-B_{0}(K) X & B_{0}(K) \\
\star & -X B_{0}(K)+D_{0}(K)
\end{array}\right)
\end{aligned}
$$

If $B_{0}(K)$ is injective, we may thus assume that $D_{0}(K)=0$, while if $B_{0}(K)$ is surjective, we may assume that $A_{0}(K)=0$. With a similar reasoning, if $B .(K)$ is injective we may assume that $D_{\bullet}(K)=0$, while if $B_{\bullet}(K)$ is surjective we may assume that $A_{\bullet}(K)=0$.

In the above decompositions for $\mathbb{H}_{\bullet}(K)$, the map $\theta(K): \mathbb{H}_{0}(K) \rightarrow \mathbb{H}_{\infty}(K)$ takes the form

$$
\theta(K)=\left(\begin{array}{cc}
X & I \\
Z & Y
\end{array}\right)
$$

since the induced map from $\mathbb{A}_{1}(K) \subset \mathbb{H}_{0}(K)$ to $\mathbb{A}_{1}(K) \subset \mathbb{H}_{\infty}(K)$ is the inverse of the map induced by $\mathfrak{f}_{1}(K)$, i.e. the identity. Moreover, since the rank of $\theta(K)$ is the same 
as the rank of $\mathfrak{f}_{1}(K)$, we conclude that $Z=Y X$. Applying the change of basis $P_{Y}$ on $\mathbb{H}_{0}(K)$ and the corresponding change of basis $P_{X}$ on $\mathbb{H}_{\infty}(K), \theta(K)$ takes the form

$$
\left(\begin{array}{cc}
I & 0 \\
-Y & I
\end{array}\right)\left(\begin{array}{cc}
X & I \\
Y X & Y
\end{array}\right)\left(\begin{array}{cc}
I & 0 \\
-X & I
\end{array}\right)=\left(\begin{array}{ll}
0 & I \\
0 & 0
\end{array}\right)
$$

It is thus possible to choose the above decompositions so that $\theta(K)=\left(\begin{array}{ll}0 & I \\ 0 & 0\end{array}\right)$. If this is the case, the $2 \times 2$ presentation of $\tau_{\infty}(K)^{-1} \bar{\theta}(K) \tau_{0}(K)$ would be of the form

$$
\tau_{\infty}(K) \bar{\theta}(K) \tau_{0}(K)=\left(\begin{array}{cc}
M & I \\
Q & P
\end{array}\right)
$$

and since the ranks of $\theta(K)$ and $\bar{\theta}(K)$ are the same, we find $Q=P M$.

\section{Subsection $5.3^{\prime}$. Simplifications of the splicing formula}

We now apply Lemma 2.3 to the splicing formula of Proposition 5.3 and make some cancellations. The first cancellation comes from setting $C=\mathbb{H}, A=\mathbb{H}_{1,1}$ and

$$
B=\left(\mathbb{H}_{\infty, \infty} \oplus \mathbb{H}_{1, \infty} \oplus \mathbb{H}_{\infty, 1}\right) \oplus\left(\mathbb{H}_{0,1} \oplus \mathbb{H}_{1,0} \oplus \mathbb{H}_{0,0}\right)=\mathbb{E}_{1} \oplus \mathbb{E}_{2}
$$

We thus have $\widehat{\mathrm{HF}}(Y)=H_{*}\left(B, d_{B}\right)$, where

$$
d_{B}=\left(\begin{array}{cccccc}
0 & \mathfrak{f}_{0}^{1} \otimes I & I \otimes \mathfrak{f}_{0}^{2} & \theta^{1} \otimes \overline{\mathfrak{f}}_{0}^{2} & \overline{\mathfrak{f}}_{0}^{1} \otimes \theta^{2} & \Gamma \\
0 & 0 & 0 & \Phi & I \otimes\left(\mathfrak{f}_{0}^{2} \circ \overline{\mathfrak{f}}_{\infty}^{2}\right) & \mathfrak{f}_{\infty}^{1} \otimes \bar{\theta}^{2} \\
0 & 0 & 0 & \left(\mathfrak{f}_{0}^{1} \circ \overline{\mathfrak{f}}_{\infty}^{1}\right) \otimes I & \Psi & \bar{\theta}^{1} \otimes \mathfrak{f}_{\infty}^{2} \\
0 & 0 & 0 & 0 & 0 & I \otimes \overline{\mathfrak{f}}_{\infty}^{2} \\
0 & 0 & 0 & 0 & 0 & \overline{\mathfrak{f}}_{\infty}^{1} \otimes I \\
0 & 0 & 0 & 0 & 0 & 0
\end{array}\right),
$$

with $\left.\Gamma=\overline{\mathfrak{f}}_{0}^{1} \circ \mathfrak{f}_{\infty}^{1}\right) \otimes \overline{\mathfrak{f}}_{0}^{2} \circ \mathfrak{f}_{\infty}^{2}+\theta^{1} \otimes \bar{\theta}^{2}+\bar{\theta}^{1} \otimes \theta^{1}, \Phi=\overline{\mathfrak{f}}_{\infty}^{1} \otimes \mathfrak{f}_{0}^{2}+\mathfrak{f}_{\infty}^{1} \otimes \overline{\mathfrak{f}}_{0}^{2}$ and $\Psi=\mathfrak{f}_{0}^{1} \otimes \overline{\mathfrak{f}}_{\infty}^{2}+\overline{\mathfrak{f}}_{0}^{1} \otimes \mathfrak{f}_{\infty}^{2}$.

The dimension of the $\mathbb{F}$-vector space $H_{*}\left(B, d_{B}\right)$ only depends on the rank of the kernel and the cokernel of the matrix $d_{B}$. Define a pair of matrices $M_{1}$ and $M_{2}$ equivalent if $\operatorname{Ker}\left(M_{1}\right) \simeq \operatorname{Ker}\left(M_{2}\right)$ and $\operatorname{Coker}\left(M_{1}\right) \simeq \operatorname{Coker}\left(M_{2}\right)$. For a matrix $M$ let

$$
\imath(M):=\operatorname{Ker}(M) \oplus \operatorname{Coker}(M) \text { and } i(M):=\operatorname{rnk}(\imath(M)) .
$$

If $M_{1}$ and $M_{2}$ are equivalent matrices then $\imath\left(M_{1}\right) \simeq \imath\left(M_{2}\right)$ and $i\left(M_{1}\right)=i\left(M_{2}\right)$. 
We make a change of basis for $\mathbb{E}_{2}$ which is given by the matrix

$$
\left(\begin{array}{ccc}
\tau_{0}\left(K_{1}\right) \otimes \tau_{1}\left(K_{2}\right) & 0 & 0 \\
0 & \tau_{1}\left(K_{1}\right) \otimes \tau_{0}\left(K_{2}\right) & 0 \\
0 & 0 & \tau_{0}\left(K_{1}\right) \otimes \tau_{0}\left(K_{2}\right)
\end{array}\right) .
$$

The matrix $d_{B}$ is thus equivalent to the matrix

$$
\begin{aligned}
d_{B}^{\prime} & =\left(\begin{array}{cccccc}
0 & \mathfrak{f}_{0}^{1} \otimes I & I \otimes \mathfrak{f}_{0}^{2} & \theta^{1} \tau_{0}^{1} \otimes \tau_{\infty}^{2} \mathfrak{f}_{0}^{2} & \tau_{\infty}^{1} \mathfrak{f}_{0}^{1} \otimes \theta^{2} \tau_{0}^{2} & \Gamma \\
0 & 0 & 0 & \Phi & \tau_{1}^{1} \otimes \mathfrak{f}_{0}^{2} \tau_{1}^{2} \mathfrak{f}_{\infty}^{2} & \mathfrak{f}_{\infty}^{1} \tau_{0}^{1} \otimes \bar{\theta}^{2} \tau_{0}^{2} \\
0 & 0 & 0 & \mathfrak{f}_{0}^{1} \tau_{1}^{1} \mathfrak{f}_{\infty}^{1} \otimes \tau_{1}^{1} & \Psi & \bar{\theta}^{1} \tau_{0}^{1} \otimes \mathfrak{f}_{\infty}^{2} \tau_{0}^{2} \\
0 & 0 & 0 & 0 & 0 & I \otimes \mathfrak{f}_{\infty}^{2} \\
0 & 0 & 0 & 0 & 0 & \mathfrak{f}_{\infty}^{1} \otimes I \\
0 & 0 & 0 & 0 & 0 & 0
\end{array}\right) . \\
\Phi & =\tau_{1}^{1} \mathfrak{f}_{\infty}^{1} \otimes \mathfrak{f}_{0}^{2} \tau_{1}^{2}+\mathfrak{f}_{\infty}^{1} \tau_{0}^{1} \otimes \tau_{\infty}^{2} \mathfrak{f}_{0}^{2}, \\
\Psi & =\mathfrak{f}_{0}^{1} \tau_{1}^{1} \otimes \tau_{1}^{2} \mathfrak{f}_{\infty}^{2}+\tau_{\infty}^{1} \mathfrak{f}_{0}^{1} \otimes \mathfrak{f}_{\infty}^{2} \tau_{0}^{2} \text { and } \\
\Gamma & =\overline{\mathfrak{f}}_{0}^{1} \mathfrak{f}_{\infty}^{1} \tau_{0}^{1} \otimes \overline{\mathfrak{f}}_{0}^{2} \mathfrak{f}_{\infty}^{2} \tau_{0}^{2}+\theta^{1} \tau_{0}^{1} \otimes \bar{\theta}^{2} \tau_{0}^{2}+\bar{\theta}^{1} \tau_{0}^{1} \otimes \theta^{2} \tau_{0}^{2}
\end{aligned}
$$

Let us use the decompositions of (5) for $K_{1}$ and $K_{2}$ to obtain a $24 \times 24$ block decomposition of $d_{B}^{\prime}$. Moreover, following the discussion at the end of Subsection 3.3 we may assume that in the corresponding decompositions,

$$
\theta^{i}=\left(\begin{array}{ll}
0 & I \\
0 & 0
\end{array}\right) \text { and }\left(\tau_{\infty}^{i}\right)^{-1} \bar{\theta}^{i} \tau_{0}^{i}=\left(\begin{array}{cc}
M^{i} & I \\
P^{i} M^{i} & P^{i}
\end{array}\right)
$$

Each entry in the above $6 \times 6$ decomposition for $d_{\mathbb{B}}^{\prime}$ corresponds to a $4 \times 4$ sub-matrix of the aforementioned $24 \times 24$ decomposition. For instance the $(1,4)$ entry $\theta^{1} \tau_{0}^{1} \otimes \tau_{\infty}^{2} f_{0}^{2}$ corresponds to

$$
\left(\begin{array}{ll}
0 & I \\
0 & 0
\end{array}\right)\left(\begin{array}{cc}
A_{0}^{1} & B_{0}^{1} \\
C_{0}^{1} & D_{0}^{1}
\end{array}\right) \otimes\left(\begin{array}{cc}
A_{\infty}^{2} & B_{\infty}^{2} \\
C_{\infty}^{2} & D_{\infty}^{2}
\end{array}\right)\left(\begin{array}{cc}
0 & 0 \\
I & 0
\end{array}\right)=\left(\begin{array}{cc}
C_{0}^{1} & D_{0}^{1} \\
0 & 0
\end{array}\right) \otimes\left(\begin{array}{cc}
B_{\infty}^{2} & 0 \\
D_{\infty}^{2} & 0
\end{array}\right)=\left(\begin{array}{cccc}
C_{0}^{1} \otimes B_{\infty}^{2} & 0 & D_{0}^{1} \otimes B_{\infty}^{2} & 0 \\
C_{0}^{1} \otimes D_{\infty}^{2} & 0 & D_{0}^{1} \otimes D_{\infty}^{2} & 0 \\
0 & 0 & 0 & 0 \\
0 & 0 & 0 & 0
\end{array}\right) .
$$

For another instance, note that the $(3,5)$ entry corresponds to

$$
\begin{aligned}
\left(\begin{array}{cccc}
0 & 0 & 0 & 0 \\
0 & 0 & 0 & 0 \\
A_{1}^{1} \otimes B_{1}^{2} & 0 & B_{1}^{1} \otimes B_{1}^{2} & 0 \\
A_{1}^{1} \otimes D_{1}^{2} & 0 & B_{1}^{1} \otimes D_{1}^{2} & 0
\end{array}\right) & +\left(\begin{array}{cccc}
0 & 0 & 0 & 0 \\
B_{\infty}^{1} \otimes A_{0}^{2} & B_{\infty}^{1} \otimes B_{0}^{2} & 0 & 0 \\
0 & 0 & 0 & 0 \\
D_{\infty}^{1} \otimes A_{0}^{2} & D_{\infty}^{1} \otimes B_{0}^{2} & 0 & 0
\end{array}\right) \\
& =\left(\begin{array}{ccccc}
0 & 0 & 0 & 0 \\
B_{\infty}^{1} \otimes A_{0}^{2} & B_{\infty}^{1} \otimes B_{0}^{2} & 0 & 0 \\
A_{1}^{1} \otimes B_{1}^{2} & 0 & B_{1}^{1} \otimes B_{1}^{2} & 0 \\
A_{1}^{1} \otimes D_{1}^{2}+D_{\infty}^{1} \otimes A_{0}^{2} & D_{\infty}^{1} \otimes B_{0}^{2} & B_{1}^{1} \otimes D_{1}^{2} & 0
\end{array}\right) .
\end{aligned}
$$

The aforementioned $24 \times 24$ decomposition includes identity matrices as the entries determined by the following block coordinates:

$(2,9),(3,5),(4,6),(14,21),(16,23)$ and $(20,22)$. 
We use the above 6 identity matrices for cancellation to obtain an equivalent matrix $d=\left(\begin{array}{cc}0 & D \\ 0 & 0\end{array}\right)$ over $\mathbb{B}_{1} \oplus \mathbb{B}_{2}$, where $\mathbb{A}_{\bullet \star}=\mathbb{A}_{\bullet}\left(K_{1}\right) \otimes \mathbb{A}_{\star}\left(K_{2}\right)$ and
\[ \mathbb{B}_{1}=\mathbb{A}_{11} \oplus \mathbb{A}_{\infty 1} \oplus \mathbb{A}_{\infty 0} \oplus \mathbb{A}_{1 \infty} \oplus \mathbb{A}_{0 \infty} \oplus \mathbb{A}_{00} \text { and } \]
$\mathbb{B}_{2}=\mathbb{A}_{\infty 0} \oplus \mathbb{A}_{10} \oplus \mathbb{A}_{\infty \infty} \oplus \mathbb{A}_{0 \infty} \oplus \mathbb{A}_{01} \oplus \mathbb{A}_{11}$.

Re-arrange the rows and the columns of the matrix $D$, so that $D$ corresponds to the rows $11,7,8,10,12,1$ and the columns $19,13,15,17,18,24$ in the above $24 \times 24$ decomposition to obtain the following matrix

$$
\left(\begin{array}{cccccc}
B_{1}^{1} \otimes B_{1}^{2} & B_{1}^{1} \otimes A_{1}^{2} & 0 & A_{1}^{1} \otimes B_{1}^{2} & 0 & 0 \\
0 & A_{0}^{1} \otimes B_{\infty}^{2} & B_{0}^{1} \otimes B_{\infty}^{2} & 0 & 0 & B_{0}^{1} \otimes\left(A_{\infty}^{2}+B_{\infty}^{2} P^{2}\right) \\
D_{1}^{1} \otimes B_{1}^{2} & D_{1}^{1} \otimes A_{1}^{2}+A_{0}^{1} \otimes D_{\infty}^{2} & B_{0}^{1} \otimes D_{\infty}^{2} & C_{1}^{1} \otimes B_{1}^{2} & 0 & B_{0}^{1} \otimes\left(C_{\infty}^{2}+D_{\infty}^{2} P^{2}\right) \\
0 & 0 & 0 & B_{\infty}^{1} \otimes A_{0}^{2} & B_{\infty}^{1} \otimes B_{0}^{2} & \left(A_{\infty}^{1}+B_{\infty}^{1} P^{1}\right) \otimes B_{0}^{2} \\
B_{1}^{1} \otimes D_{1}^{2} & B_{1}^{1} \otimes C_{1}^{2} & 0 & D_{\infty}^{1} \otimes A_{0}^{2}+A_{1}^{1} \otimes D_{1}^{2} & D_{\infty}^{1} \otimes B_{0}^{2} & \left(C_{\infty}^{1}+D_{\infty}^{1} P^{1}\right) \otimes B_{0}^{2} \\
0 & C_{0}^{1} \otimes B_{\infty}^{2} & D_{0}^{1} \otimes B_{\infty}^{2} & B_{\infty}^{1} \otimes C_{0}^{2} & B_{\infty}^{1} \otimes D_{0}^{2} & \Gamma
\end{array}\right)
$$

with

$$
\Gamma=B_{\infty}^{1} \bar{B}_{1}^{1} B_{0}^{1} \otimes B_{\infty}^{2} \bar{B}_{1}^{2} B_{0}^{2}+\left(A_{\infty}^{1}+B_{\infty}^{1} P^{1}\right) \otimes D_{0}^{2}+D_{0}^{1} \otimes\left(A_{\infty}^{2}+B_{\infty}^{2} P^{2}\right) .
$$

Here, we assume that

$$
\left(\tau_{\bullet}^{i}\right)^{-1}=\left(\begin{array}{ll}
\bar{A}_{\bullet}^{i} & \bar{B}_{\bullet}^{i} \\
\bar{C}_{\bullet}^{i} & \bar{D}_{\bullet}^{i}
\end{array}\right), \quad \bullet \in\{0,1, \infty\}, i=1,2,
$$

are the corresponding block decompositions for the inverses of $\tau_{\bullet}^{i}$.

This matrix is in turn equivalent to the matrix $\mathfrak{D}=\mathfrak{D}\left(K_{1}, K_{2}\right)$ below, which is obtained by adding $I \otimes P^{2}$ times the third column and $P^{1} \otimes I$ times the fifth column to the last column of the above matrix:

$$
\mathfrak{D}=\left(\begin{array}{cccccc}
B_{1}^{1} \otimes B_{1}^{2} & C_{1}^{1} \otimes A_{1}^{2} & 0 & A_{1}^{1} \otimes B_{1}^{2} & 0 & 0 \\
0 & A_{0}^{1} \otimes B_{\infty}^{2} & B_{0}^{1} \otimes B_{\infty}^{2} & 0 & 0 & B_{0}^{1} \otimes A_{\infty}^{2} \\
D_{1}^{1} \otimes B_{1}^{2} & D_{1}^{1} \otimes A_{1}^{2}+A_{0}^{1} \otimes D_{\infty}^{2} & B_{0}^{1} \otimes D_{\infty}^{2} & C_{1}^{1} \otimes B_{1}^{2} & 0 & B_{0}^{1} \otimes C_{\infty}^{2} \\
0 & 0 & 0 & B_{\infty}^{1} \otimes A_{0}^{2} & B_{\infty}^{1} \otimes B_{0}^{2} & A_{\infty}^{1} \otimes B_{0}^{2} \\
B_{1}^{1} \otimes D_{1}^{2} & B_{1}^{1} \otimes C_{1}^{2} & 0 & D_{\infty}^{1} \otimes A_{0}^{2}+A_{1}^{1} \otimes D_{1}^{2} & D_{\infty}^{1} \otimes B_{0}^{2} & C_{\infty}^{1} \otimes B_{0}^{2} \\
0 & C_{0}^{1} \otimes B_{\infty}^{2} & D_{0}^{1} \otimes B_{\infty}^{2} & B_{\infty}^{1} \otimes C_{0}^{2} & B_{\infty}^{1} \otimes D_{0}^{2} & \Psi
\end{array}\right),
$$

where $\Psi=A_{\infty}^{1} \otimes D_{0}^{2}+D_{0}^{1} \otimes A_{\infty}^{2}+X^{1} \otimes X^{2}$ and $X^{i}=X\left(K_{i}\right)=B_{\infty}^{i} \bar{B}_{1}^{i} B_{0}^{i}$ for $i=1,2$.

Combining Proposition 5.3 with the above observations we find: 
Proposition 5.5 Let $K_{i} \subset Y_{i}$ denote null-homologous knots for $i=1,2$ and $Y\left(K_{1}, K_{2}\right)$ denote the three-manifold obtained by splicing the complement of $K_{1}$ with the complement of $K_{2}$. With the above definition of $\mathfrak{D}\left(K_{1}, K_{2}\right)$

$$
\widehat{\mathrm{HF}}\left(Y\left(K_{1}, K_{2}\right), \mathbb{F}\right) \simeq \imath\left(\mathfrak{D}\left(K_{1}, K_{2}\right)\right)
$$

Corollary 5.6 The splicing formula of Proposition 5.3 is independent of the choice of extensions $\theta^{i}$ and $\bar{\theta}^{i}$.

Proof. The fact that the matrices $P^{i}$ and $M^{i}$ do not appear in the matrix $\mathfrak{D}\left(K_{1}, K_{2}\right)$ implies that the choice of the extensions $\theta^{i}, \bar{\theta}^{i}: \mathbb{H}_{0}^{i} \rightarrow \mathbb{H}_{\infty}^{i}$ does not change the rank of the homology group in the splicing formula of Proposition 5.3 or Theorem 1.1.

With the above corollary in place, the proof of Theorem 1.1 is now complete.

Definition 5.7 For a pair of knots $K_{i} \subset Y_{i}, i=1,2$ define

$$
\begin{aligned}
\chi\left(K_{1}, K_{2}\right):=\left(h_{1}\left(K_{1}\right)-h_{\infty}\left(K_{1}\right)\right)\left(h_{1}\left(K_{2}\right)-h_{\infty}\left(K_{2}\right)\right) \\
-\left(h_{1}\left(K_{1}\right)-h_{0}\left(K_{1}\right)\right)\left(h_{1}\left(K_{2}\right)-h_{0}\left(K_{2}\right)\right)
\end{aligned}
$$

Note that $\chi\left(K_{1}, K_{2}\right)$ is in fact the difference between the ranks of $\mathbb{B}_{1}=\mathbb{B}_{1}\left(K_{1}, K_{2}\right)$ and $\mathbb{B}_{2}=\mathbb{B}_{2}\left(K_{1}, K_{2}\right)$. In the corresponding $\mathbb{Z} / 2 \mathbb{Z}$-grading on $\mathbb{B}_{1} \oplus \mathbb{B}_{2}, \chi\left(K_{1}, K_{2}\right)$ is thus the Euler characteristic of the chain complex $\left(\mathbb{B}_{1} \oplus \mathbb{B}_{2}, d\right)$.

Corollary 5.8 With the above notation fixed,

$$
\operatorname{rnk}\left(\widehat{\operatorname{HF}}\left(Y\left(K_{1}, K_{2}\right)\right)\right) \geq\left|\chi\left(K_{1}, K_{2}\right)\right| .
$$

Proof. It is enough to note that

$$
\chi\left(K_{1}, K_{2}\right)=\operatorname{rnk}\left(\operatorname{Ker}\left(\mathfrak{D}\left(K_{1}, K_{2}\right)\right)\right)-\operatorname{rnk}\left(\operatorname{Coker}\left(\mathfrak{D}\left(K_{1}, K_{2}\right)\right)\right) .
$$


Consider the matrices

$$
P_{L}=\left(\begin{array}{cccccc}
I \otimes \bar{A}_{1}^{2} & 0 & 0 & 0 & I \otimes \bar{B}_{1}^{2} & 0 \\
0 & I \otimes \bar{A}_{\infty}^{2} & I \otimes \bar{B}_{\infty}^{2} & 0 & 0 & 0 \\
0 & I \otimes \bar{C}_{\infty}^{2} & I \otimes \bar{D}_{\infty}^{2} & 0 & 0 & 0 \\
0 & 0 & 0 & I \otimes \bar{A}_{0}^{2} & 0 & I \otimes \bar{B}_{0}^{2} \\
I \otimes \bar{C}_{1}^{2} & 0 & 0 & 0 & I \otimes \bar{D}_{1}^{2} & 0 \\
0 & 0 & 0 & I \otimes \bar{C}_{0}^{2} & 0 & I \otimes \bar{D}_{0}^{2}
\end{array}\right)
$$

and

$$
P_{R}=\left(\begin{array}{cccccc}
\bar{D}_{1}^{1} \otimes I & 0 & 0 & \bar{C}_{1}^{1} \otimes I & 0 & 0 \\
0 & \bar{A}_{0}^{1} \otimes I & \bar{B}_{0}^{1} \otimes I & 0 & 0 & 0 \\
0 & \bar{C}_{0}^{1} \otimes I & \bar{D}_{0}^{1} \otimes I & 0 & 0 & 0 \\
\bar{B}_{1}^{1} \otimes I & 0 & 0 & \bar{A}_{1}^{1} \otimes I & 0 & 0 \\
0 & 0 & 0 & 0 & \bar{D}_{\infty}^{1} \otimes I & \bar{C}_{\infty}^{1} \otimes I \\
0 & 0 & 0 & 0 & \bar{B}_{\infty}^{1} \otimes I & \bar{A}_{\infty}^{1} \otimes I
\end{array}\right) .
$$

Both $P_{R}$ and $P_{L}$ are invertible and $\mathfrak{D}\left(K_{1}, K_{2}\right)$ is equivalent to $\mathfrak{D}^{\prime}\left(K_{1}, K_{2}\right)=P_{L} \mathfrak{D}\left(K_{1}, K_{2}\right) P_{R}$.

The matrix $\mathfrak{D}^{\prime}\left(K_{1}, K_{2}\right)$ has the following block presentation.

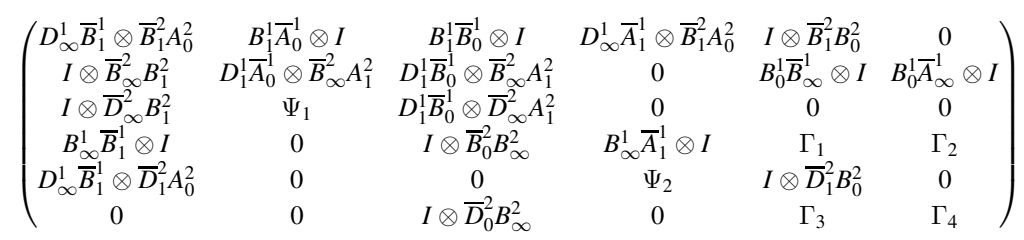

with

$$
\begin{aligned}
& \Psi_{1}=I \otimes I+D_{1}^{1} \bar{A}_{0}^{1} \otimes \bar{D}_{\infty}^{2} A_{1}^{2} \\
& \Psi_{2}=I \otimes I+D_{\infty}^{1} \bar{A}_{1}^{1} \otimes \bar{D}_{1}^{2} A_{0}^{2} \\
& \Gamma_{1}=D_{0}^{1} \bar{B}_{\infty}^{1} \otimes \bar{B}_{0}^{2} A_{\infty}^{2}+X^{1} \bar{B}_{\infty}^{1} \otimes \bar{B}_{0}^{2} X^{2} \\
& \Gamma_{2}=D_{0}^{1} \bar{A}_{\infty}^{1} \otimes \bar{B}_{0}^{2} A_{\infty}^{2}+X^{1} \bar{A}_{\infty}^{1} \otimes \bar{B}_{0}^{2} X^{2} \\
& \Gamma_{3}=D_{0}^{1} \bar{B}_{\infty}^{1} \otimes \bar{D}_{0}^{2} A_{\infty}^{2}+X^{1} \bar{B}_{\infty}^{1} \otimes \bar{D}_{0}^{2} X^{2} \\
& \Gamma_{4}=I \otimes I+D_{0}^{1} \bar{A}_{\infty}^{1} \otimes \bar{D}_{0}^{2} A_{\infty}^{2}+X^{1} \bar{A}_{\infty}^{1} \otimes \bar{D}_{0}^{2} X^{2},
\end{aligned}
$$

and is easier to use in actual computations. Note that

$$
\imath\left(\mathfrak{D}^{\prime}\left(K_{1}, K_{2}\right)\right) \simeq \imath\left(\mathfrak{D}\left(K_{1}, K_{2}\right)\right) \simeq \widehat{\mathrm{HF}}\left(Y\left(K_{1}, K_{2}\right), \mathbb{F}\right) .
$$

\section{Subsection $6.2^{\prime}$. Splicing a knot complement with the complement of a trefoil}

For a knot $K \subset Y$, let $Y(R, K)$ denote the three-manifold obtained by splicing the complement of $K \subset Y$ with the complements of the right handed trefoil. We study 
the rank $r_{r}(K)$ of $\widehat{\mathrm{HF}}(Y(R, K))$ in this subsection. With the notation of Subsection , $r_{r}(K)=i\left(\mathfrak{D}^{\prime}(R, K)\right)$. Replacing the block forms of $(21)$ in $\mathfrak{D}^{\prime}(R, K)$, we find

$$
\mathfrak{D}^{\prime}(R, K)=\left(\begin{array}{cccccccccc}
0 & 0 & 0 & 0 & 0 & I & 0 & \bar{B}_{1} B_{0} & 0 & 0 \\
\bar{B}_{\infty} B_{1} & 0 & 0 & 0 & 0 & 0 & 0 & 0 & 0 & I \\
0 & \bar{B}_{\infty} B_{1} & 0 & 0 & \bar{B}_{\infty} A_{1} & 0 & 0 & I & 0 & 0 \\
\bar{D}_{\infty} B_{1} & 0 & I & 0 & 0 & 0 & 0 & 0 & 0 & 0 \\
0 & \bar{D}_{\infty} B_{1} & 0 & I & \bar{D}_{\infty} A_{1} & 0 & 0 & 0 & 0 & 0 \\
I & 0 & 0 & 0 & \bar{B}_{0} B_{\infty} & 0 & 0 & 0 & 0 & \bar{B}_{0} X \\
0 & 0 & 0 & 0 & 0 & \bar{B}_{0} B_{\infty} & 0 & 0 & 0 & 0 \\
0 & 0 & 0 & 0 & 0 & 0 & I & \bar{D}_{1} B_{0} & 0 & 0 \\
0 & 0 & 0 & 0 & \bar{D}_{0} B_{\infty} & 0 & 0 & 0 & I & \bar{D}_{0} X \\
0 & 0 & 0 & 0 & 0 & \bar{D}_{0} B_{\infty} & 0 & 0 & 0 & I
\end{array}\right),
$$

where $A_{\bullet}=A_{\bullet}(K), B_{\bullet}=B_{\bullet}(K), C_{\bullet}=C_{\bullet}(K), D_{\bullet}=D_{\bullet}(K), \bar{A}_{\bullet}=\bar{A}_{\bullet}(K), \bar{B}_{\bullet}=$ $\bar{B} \cdot(K), \bar{C} \bullet=\bar{C}_{\bullet}(K), \bar{D} \bullet=\bar{D}_{\bullet}(K)$ and $X=X(K)$ for $\bullet \in\{0,1, \infty\}$.Doing a series of cancellations that correspond to the identity matrices which appear as the

$$
(1,6),(3,8),(4,3),(5,4),(6,1),(8,7),(9,9) \text { and }(10,10)
$$

entries in the above block presentation we obtain the equivalent matrix

$$
R_{r}(K):=\left(\begin{array}{cc}
0 & \bar{B}_{0} X \bar{B}_{\infty} \\
\bar{X} B_{\infty} \bar{B}_{1} & \bar{X} B_{\infty} \bar{A}_{1}+\bar{D}_{0} X \bar{B}_{\infty}
\end{array}\right)
$$

Where $\bar{X}=\bar{X}(R)=\bar{B}_{\infty}(R) B_{1}(R) \bar{B}_{0}(R)$.

Corollary 6.1 For a knot $K \subset Y$ let $Y(R, K)$ denote the three-manifold obtained by splicing the complement of $K$ and the complement of the trefoil. Then

$$
\widehat{\mathrm{HF}}(Y(R, K))=\imath\left(R_{r}(K)\right) .
$$

Proof. The claim follows immediately from the above discussion.

For the trefoils, our computations imply that

$$
\begin{aligned}
& \bar{X}(R) B_{\infty}(R)=X(R) \bar{B}_{\infty}(R)=\bar{X}(L) B_{\infty}(L)=X(L) \bar{B}_{\infty}(L)=0 \\
\Rightarrow & R_{r}(R)=R_{r}(L)=0 \\
\Rightarrow & |\widehat{\mathrm{HF}}(Y(R, R))|=7 \text { and }|\widehat{\mathrm{HF}}(Y(R, L))|=9 .
\end{aligned}
$$

The above computations agree with the computations of Hedden and Levine [3].

Corollary 6.2 For every knot $K$ in a homology sphere $Y$ we have

$$
\begin{aligned}
|\widehat{\mathrm{HF}}(Y(R, K))| & \geq\left(a_{0}(K)+a_{1}(K)+2 a_{\infty}(K)\right)-4 \min \left\{a_{0}(K), a_{1}(K), a_{\infty}(K)\right\} \\
& =4 \max \left\{h_{0}(K), h_{1}(K), h_{\infty}(K)\right\}-\left(h_{0}(K)+h_{1}(K)+2 h_{\infty}(K)\right) .
\end{aligned}
$$


Moreover, if $Y(R, K)$ is a homology sphere $L$-space $K$ is trivial and $Y$ is a homology sphere $L$-space.

Proof. Let $M=M(K)=X(K) \bar{B}_{\infty}(K)$ and $\bar{M}=\bar{M}(K)=\bar{X}(K) B_{\infty}(K)$ and note that

$$
\begin{aligned}
& \operatorname{rnk}\left(R_{r}(K)\right)=\operatorname{rnk}\left(\begin{array}{cc}
0 & \bar{B}_{0}(K) M \\
\bar{M} B_{1}(K) & \bar{M} A_{1}(K)+\bar{D}_{0}(K) M
\end{array}\right)
\end{aligned}
$$

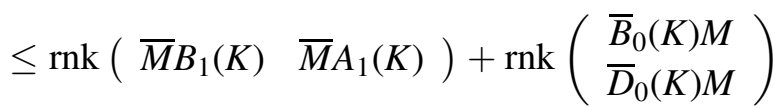

$$
\begin{aligned}
& =\operatorname{rnk}(M)+\operatorname{rnk}(\bar{M}) .
\end{aligned}
$$

For every knot $K \subset Y$ as above note that the ranks of $M=M(K)$ and $\bar{M}=\bar{M}(K)$ are at most equal to the minimum of the sizes of the matrices $B_{0}(K), B_{1}(K)$ and $B_{\infty}(K)$, which is

$$
\min \left\{a_{0}(K), a_{1}(K), a_{\infty}(K)\right\} .
$$

Since $R_{r}(K)$ is of size $h_{0}(K) \times h_{1}(K)=\left(a_{1}(K)+a_{\infty}(K)\right) \times\left(a_{0}(K)+a_{\infty}(K)\right.$ this proves the first part of the corollary.

Let us assume that $\operatorname{rnk}(\widehat{\mathrm{HF}}(Y(R, K)))=1$. From here we find

$$
\begin{aligned}
\left(a_{0}(K)+\right. & \left.a_{1}(K)+2 a_{\infty}(K)\right)-4 \min \left\{a_{0}(K), a_{1}(K), a_{\infty}(K)\right\} \\
& =\left(a_{0}(K)+a_{1}(K)+2 a_{\infty}(K)\right)-2 \operatorname{rnk}(M)-2 \operatorname{rnk}(\bar{M})=1 .
\end{aligned}
$$

Since $a_{1}(K)$ and $a_{\infty}(K)$ have the same parity while the parity of $a_{0}(K)$ is different from the parity of both $a_{1}(K)$ and $a_{\infty}(K)$, one can easily conclude that $a_{0}(K)-1=$ $a_{1}(K)=a_{\infty}(K)$. Let $a$ denote the common value $a_{1}(K)=a_{\infty}(K)$. Then the ranks of $M$ and $\bar{M}$ are $a$ and $B_{0}(K), X(K)$ and $\bar{X}(K)$ are all invertible. We may thus assume that $A_{0}(K)=D_{0}(K)=0$. Since

$$
\operatorname{rnk}\left(\mathfrak{f}_{\infty}(K)+\overline{\mathfrak{f}}_{\infty}(K)\right)=\operatorname{rnk}\left(\begin{array}{cc}
B_{1}(K) A_{0}(K) & B_{1}(K) B_{0}(K) \\
I+D_{1}(K) A_{0}(K) & D_{1}(K) B_{0}(K)
\end{array}\right)=2 a
$$

the three-manifold $Y$ is an $L$-space. Since splicing $K$ with the trefoil is also a homology sphere $L$-space we conclude that $K$ is trivial, by Theorem 1 from [3].

\section{Appendix'; Bordered Floer homology for knot complements}

The first draft of this paper appeared while the theory of bordered Floer homology was being developed. With bordered Floer homology conventions widely known to 
the Heegaard Floer community, the referee recommended the inclusion of an appendix which addresses the contribution of this paper within the realm of bordered Floer homology.

Let $K \subset Y$ denote a null-homologous knot inside the three-manifold $Y$, and let $H=(\Sigma, \boldsymbol{\alpha}, \widehat{\boldsymbol{\beta}} \cup\{\lambda, \mu\} ; z)$ denote a special Heegaard diagram for $K$, as constructed in Lemma 4.1. In particular, $H$ is a nice Heegaard diagram for the bordered three-manifold $Y_{K}$ determined by $K \subset Y$ in the sense of [5]. The Bordered Floer complex $\widehat{\mathrm{CFD}}\left(Y_{K}\right)$ may then be constructed from the chain complexes $M=M(K)$ and $L=L(K)$ (which are described in Proposition 5.1 as the mapping cones of $\overline{\mathfrak{f}}_{\infty}(K): C_{0}(K) \rightarrow C_{1}(K)$ and $\mathfrak{f}_{0}(K): C_{1}(K) \rightarrow C_{\infty}(K)$, respectively) and the chain maps $\Phi=\Phi(K): L \rightarrow M$ and $\Psi_{i}=\Psi_{i}(K): M \rightarrow L, i=1,2,3$.

More precisely and following the notation of Subsection 4.2 from [6], the idempotents $\imath_{0}$ and $\imath_{1}$, and the chords $\rho_{1}, \rho_{2}, \rho_{3}, \rho_{12}=\rho_{1} \rho_{2}, \rho_{23}=\rho_{2} \rho_{3}$ and $\rho_{123}=\rho_{1} \rho_{2} \rho_{3}$ form a $\mathbb{F}$-basis for the differential graded algebra associated with the torus boundary;

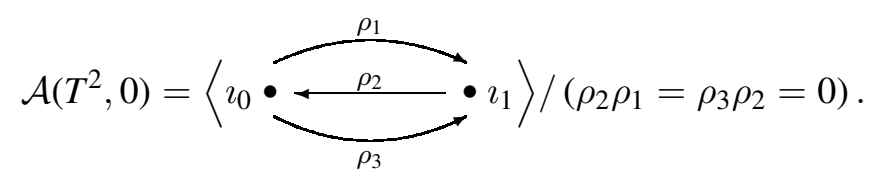

The module $\widehat{\operatorname{CFD}}\left(Y_{K}\right)$ is generated (over $\mathcal{A}\left(T^{2}, 0\right)$ ) by the generators of $M$ and $L$. For a generator $\mathbf{x}$ of $L$ we have

$$
I(\mathbf{x})=\iota_{0} \quad \text { and } \quad \partial(\mathbf{x})=d_{L}(\mathbf{x})+\rho_{1} \Psi_{1}(\mathbf{x})+\rho_{3} \Psi_{2}(\mathbf{x})+\rho_{123} \Psi_{3}(\mathbf{x}),
$$

while for a generator $\mathbf{y}$ of $M$ we have

$$
I(\mathbf{y})=\imath_{1} \quad \text { and } \quad \partial(\mathbf{y})=d_{M}(\mathbf{y})+\rho_{2} \Phi(\mathbf{y}) .
$$

The splicing formula of (17) is then just the gluing formula for bordered Floer homology, i.e. Theorem 1.3 from [5]. A related discussion is carried over in Section 8 of [5].

Definition A.1 The chain complexes $\left(C_{\bullet}(K), d_{\bullet}\right), \bullet \in\{0,1, \infty\}$ and the chain maps $f_{\bullet}(K), \bar{f} .(K), \bullet \in\{0, \infty\}$ are called admissible data associated with the knot $K$ if they satisfy the following conditions:

- The homology of the complex $\left(C_{\bullet}(K), d_{\bullet}\right)$ is $\mathbb{H}_{\bullet}(K)$. 
- The maps induced by $f_{\bullet}(K)$ and $\bar{f}$. $(K)$ in homology (under the identification of the homology of $\left(C_{\bullet}(K), d_{\bullet}\right)$ with $\left.\mathbb{H}_{\bullet}(K)\right)$ are $\mathfrak{f}_{\bullet}(K)$ and $\overline{\mathfrak{f}}_{\bullet}(K)$, respectively.

- We have $f_{0}(K) \circ f_{\infty}(K)=\bar{f}_{0}(K) \circ \bar{f}_{\infty}(K)=0$.

- The corresponding maps $\theta(K): \operatorname{Ker}\left(\mathfrak{f}_{\infty}(K)\right) \rightarrow \operatorname{Coker}\left(\mathfrak{f}_{0}(K)\right)$ and $\bar{\theta}(K): \operatorname{Ker}\left(\overline{\mathfrak{f}}_{\infty}(K)\right) \rightarrow \operatorname{Coker}\left(\overline{\mathfrak{f}}_{0}(K)\right)$ are isomorphisms, and are the inverses of the maps induces by $\mathfrak{f}_{1}(K)$ and $\overline{\mathfrak{f}}_{1}(K)$, respectively.

The proof of Theorem 1.1 implies that $\left(C_{\bullet}^{i}, d_{\bullet}^{i}\right)$ and the chain maps $f_{\bullet}^{i}, \bar{f}_{\bullet}^{i}$ for $\bullet \in$ $\{0, \infty\}, i=1,2$ in (17) may be replaced by other admissible data corresponding to the knots $K_{1}$ and $K_{2}$. Correspondingly, the bordered Floer complex associated with any knot $K \subset Y$ may be constructed from admissible data associated with $K$. More precisely, we have the following proposition.

Proposition A.2 Suppose that the chain complexes $\left(C_{\bullet}(K), d_{\bullet}\right), \bullet \in\{0,1, \infty\}$ and the chain maps $f_{\bullet}=f_{\bullet}(K), \bar{f}_{\bullet}=\bar{f}_{\bullet}(K), \bullet \in\{0, \infty\}$ are admissible data associated with the knot $K \subset Y$ and set

$$
M(K)=C_{0}(K) \oplus C_{1}(K), L(K)=C_{1}(K) \oplus C_{\infty}(K)
$$

The bordered Floer complex $\widehat{\mathrm{CFD}}\left(Y_{K}\right)$ may then be constructed as the left module over the differential graded algebra $\mathcal{A}\left(T^{2}, 0\right)$ which is generated by $\imath_{0} . L(K)$ and $\imath_{1} . M(K)$, and equipped with the differential $\partial: \widehat{\operatorname{CFD}}\left(Y_{K}\right) \rightarrow \widehat{\operatorname{CFD}}\left(Y_{K}\right)$ defined by

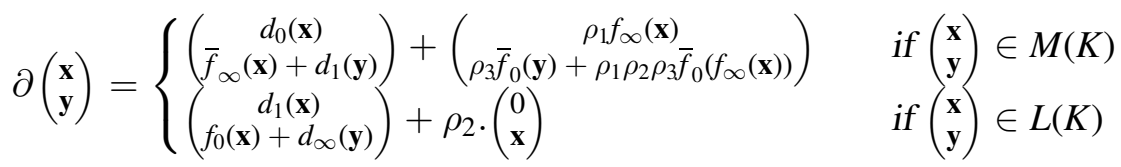

In particular, let the $\mathbb{F}$-modules $\mathbb{A}_{\bullet}=\mathbb{A}_{\bullet}(K), \bullet\{0,1, \infty\}$ and the matrices $A_{\bullet}=$ $A_{\bullet}(K), B_{\bullet}=B_{\bullet}(K), C_{\bullet}=C_{\bullet}(K)$ and $D_{\bullet}=D_{\bullet}(K)$ be defined as in Subsection 3.3. Set

$$
\begin{aligned}
& \left(C_{0}(K), d_{0}\right)=\left(\mathbb{A}_{\infty} \oplus \mathbb{A}_{1}, 0\right), \quad\left(C_{\infty}(K), d_{\infty}\right)=\left(\mathbb{A}_{1} \oplus \mathbb{A}_{0}, 0\right) \\
& C_{1}(K)=\mathbb{A}_{1} \oplus \mathbb{A}_{0} \oplus \mathbb{A}_{\infty} \oplus \mathbb{A}_{1} \quad \text { and } d_{1}=\left(\begin{array}{cccc}
0 & 0 & 0 & 0 \\
0 & 0 & 0 & 0 \\
0 & 0 & 0 & 0 \\
I_{\mathbb{A}_{1}} & 0 & 0 & 0
\end{array}\right) .
\end{aligned}
$$


Correspondingly, define

$$
\begin{aligned}
& f_{\infty}(K)=\left(\begin{array}{ll}
0 & 0 \\
0 & 0 \\
I & 0 \\
0 & I
\end{array}\right), \quad f_{0}(K)=\left(\begin{array}{cccc}
I & 0 & 0 & 0 \\
0 & I & 0 & 0
\end{array}\right), \\
& \tau_{1}(K)=\left(\begin{array}{cccc}
0 & 0 & 0 & 0 \\
0 & A_{1} & B_{1} & 0 \\
0 & C_{1} & D_{1} & 0 \\
0 & 0 & 0 & 0
\end{array}\right), \quad \bar{\tau}_{1}(K)=\left(\begin{array}{ccccc}
0 & 0 & 0 & 0 \\
0 & \bar{A}_{1} & \bar{B}_{1} & 0 \\
0 & \bar{C}_{1} & \bar{D}_{1} & 0 \\
0 & 0 & 0 & 0
\end{array}\right)
\end{aligned}
$$

and set $\bar{f}_{\infty}(K)=\tau_{1}(K) f_{\infty}(K) \tau_{0}^{-1}(K)$ and $\bar{f}_{0}(K)=\tau_{\infty}(K) f_{0}(K) \bar{\tau}_{1}(K)$.The data associated with $K$ consisting of $\left(C_{\bullet}(K), d_{\bullet}\right)$ and $f_{\bullet}(K), \bar{f}_{\bullet}(K), \bullet \in\{0, \infty\}$ is then admissible.

Corresponding to the above admissible data and associated with $K \subset Y$ we may construct the bordered Floer complex for $K$ via

$$
\begin{aligned}
& M(K)=C_{0}(K) \oplus C_{1}(K)=\mathbb{A}_{\infty} \oplus \mathbb{A}_{1} \oplus \mathbb{A}_{1} \oplus \mathbb{A}_{0} \oplus \mathbb{A}_{\infty} \oplus \mathbb{A}_{1} \\
& L(K)=C_{1}(K) \oplus C_{\infty}(K)=\mathbb{A}_{1} \oplus \mathbb{A}_{0} \oplus \mathbb{A}_{\infty} \oplus \mathbb{A}_{1} \oplus \mathbb{A}_{1} \oplus \mathbb{A}_{0} \\
& d_{M}=\left(\begin{array}{cccccc}
0 & 0 & 0 & 0 & 0 & 0 \\
0 & 0 & 0 & 0 & 0 & 0 \\
0 & 0 & 0 & 0 & 0 & 0 \\
B_{1} \bar{A}_{0} & B_{1} \bar{B}_{0} & 0 & 0 & 0 & 0 \\
D_{1} \bar{A}_{0} & D_{1} \bar{B}_{0} & I & 0 & 0 & 0 \\
0 & 0 & 0 & 0 & 0 & 0
\end{array}\right), \quad d_{L}=\left(\begin{array}{cccccc}
0 & 0 & 0 & 0 & 0 & 0 \\
0 & 0 & 0 & 0 & 0 & 0 \\
0 & 0 & 0 & 0 & 0 & 0 \\
I & 0 & 0 & 0 & 0 & 0 \\
0 & 0 & I & 0 & 0 & 0 \\
0 & 0 & 0 & I & 0 & 0
\end{array}\right) \\
& \Phi(K)=\left(\begin{array}{cccccc}
0 & 0 & 0 & 0 & 0 & 0 \\
0 & 0 & 0 & 0 & 0 & 0 \\
I & 0 & 0 & 0 & 0 & 0 \\
0 & I & 0 & 0 & 0 & 0 \\
0 & 0 & I & 0 & 0 & 0 \\
0 & 0 & 0 & I & 0 & 0
\end{array}\right), \quad \Psi_{1}(K)=\left(\begin{array}{cccccc}
0 & 0 & 0 & 0 & 0 & 0 \\
0 & 0 & 0 & 0 & 0 & 0 \\
I & 0 & 0 & 0 & 0 & 0 \\
0 & I & 0 & 0 & 0 & 0 \\
0 & 0 & 0 & 0 & 0 & 0 \\
0 & 0 & 0 & 0 & 0 & 0
\end{array}\right) \\
& \Psi_{2}(K)=\left(\begin{array}{cccccc}
0 & 0 & 0 & 0 & 0 & 0 \\
0 & 0 & 0 & 0 & 0 & 0 \\
0 & 0 & 0 & 0 & 0 & 0 \\
0 & 0 & 0 & 0 & 0 & 0 \\
0 & 0 & 0 & B_{\infty} \bar{A}_{1} & B_{\infty} \bar{B}_{1} & 0 \\
0 & 0 & 0 & D_{\infty} \bar{A}_{1} & D_{\infty} \bar{B}_{1} & 0
\end{array}\right) \text { and } \Psi_{3}(K)=\Psi_{2}(K) \Phi(K) \Psi_{1}(K) .
\end{aligned}
$$


as the left module over the differential graded algebra $\mathcal{A}\left(T^{2}, 0\right)$ generated by $\imath_{0} . L$ and $\imath_{1} . M$ and equipped with the differential $\partial: \widehat{\operatorname{CFD}}\left(Y_{K}\right) \rightarrow \widehat{\operatorname{CFD}}\left(Y_{K}\right)$ defined by the equations (24) and (25).

Remark A.3 Simultaneous computation of the matrices $\tau_{\bullet}(K)=\left(\begin{array}{cc}A_{\bullet} & B \bullet \\ C_{\bullet} & D_{\bullet}\end{array}\right)$ is a priori quite difficult, as we observed in the case of trefoils in Section 6. This makes the above description of the bordered Floer homology hard to use even for knots $K \subset Y$ where we have complete understanding of the Heegaard Floer complex associated with $K$. However, it is possible to construct admissible data associated with $K \subset Y$ completely in terms of the filtered chain complex $\mathrm{CF}^{\infty}(Y, K ; \mathbb{F})$, as will be discussed in the revision of [2].

\section{References}

[1] Eftekhary, E., Floer homology and splicing knot complements, Algebr. Geom. Topol. 15 (2015), no. 6, 3155â^^3213.

[2] Eftekhary, E., Bordered Floer homology and existence of incompressible tori in homology spheres, to appear in Compos. Math.

[3] Hedden, M., Levine, A. S., Splicing knot complements and bordered Floer homology, to appear in J. Reine Angew. Math., available at arXiv:1210.7055.

[4] Hendricks, K., Manolescu, C., Involutive Heegaard Floer homology, preprint, available at arXiv: 1507.00383.

[5] Lipshitz, R., Ozsváth, O., Thurston, D., Bordered Heegaard Floer homology: Invariance and pairing, preprint, available at arXiv:0810.0687.

[6] Lipshitz, R., Ozsvath, O., Thurston, D., Notes on bordered Floer homology, Contact and symplectic topology, 275-355, Bolyai Soc. Math. Stud., 26, János Bolyai Math. Soc., Budapest, 2014.

[7] Sarkar, S., Moving basepoints and the induced automorphisms of link Floer homology, Algebr. Geom. Topol., 15(2015), no. 5, 2479-2515.

School of Mathematics, Institute for Research in Fundamental Sciences (IPM), P. O. Box 19395-5746, Tehran, Iran

eaman@ipm.ir

http://math.ipm.ir/ eftekhary 